\title{
REAPROPIÁNDONOS DE NUESTRAS VIDAS. UN ESPACIO PARA EL EMPODERAMIENTO Y LA VISIBILIZACIÓN DE MUJERES LESBIANAS, BISEXUALES Y PANSEXUALES
}

\author{
Andrea Francisco \\ Universitat Jaume I (UJI)
}

Laura Poch Riquer

Asociación Enruta’t

RESUMEN: El presente artículo busca difundir el proyecto Reapropiándonos de nuestras vidas. En él, un grupo de mujeres lesbianas, bisexuales y pansexuales elaboramos colaborativamente relatos de nuestras vidas con el objetivo de visibilizar experiencias fuera de los discursos producidos por el sistema patriarcal y heteronormativo. En el proceso construimos un espacio de seguridad, confianza e intimidad que nos ha permitido repensar nuestra vida junto con otras, además de generar un material didáctico para trabajar la diversidad sexual a partir de mostrar modelos, prácticas y deseos alternativos a la heterosexualidad.

Palabras clave: diversidad sexual, historias de vida, empoderamiento, LGTBIQ+.

RESUM: Aquest article busca difondre el projecte Reapropiant-nos de les nostres vides. En aquest, un grup de dones lesbianes, bisexuals i pansexuals elaborem col-laborativament relats de les nostres vides amb l'objectiu de visibilitzar experiències fora dels discursos produïts pel sistema patriarcal i heteronormatiu. En el procés construïm un espai de seguretat, confiança i intimitat que ens ha permès repensar la nostra vida al costat d'altres, a més de generar un material didàctic per a treballar la diversitat sexual a partir de mostrar models, pràctiques i desitjos alternatius a l'heterosexualitat.

Paraules Clau: diversitat sexual, històries de vida, apoderament, LGTBiQ+. 
AвSTRACT: This article seeks to spread the project Reapropiándonos de nuestras vidas. In it, a group of lesbians, bisexual and pansexual collaboratively elaborate stories of our lives in order to make visible practices outside the discourses produced by the patriarchal and heteronormative system. In the process we construct a space of safety, trust and intimacy that allowed us think our life with others, and generate a didactic material to work with sexual diversity by showing models, practices and desires alternative to heterosexuality.

KeYWORDS: sexual diversity, life histories, empowerment, LGTBIQ+.

\section{Una reflexión inicial: cuerpos disidentes en el espacio público}

\section{$\mathrm{L}$} a vida requiere que social y políticamente se cumplan condiciones que le permitan mantenerse. Si una vida no tiene elementos para sostenerse, si social y políticamente dicha vida ha sido arrojada al aislamiento, podemos señalar que su vulnerabilidad no ha sido reconocida, que dicha vida no es entendida como vida habitable (Castro, 2014). Vemos cómo la vida de algunos se cuida más que las de otros, como la vulnerabilidad originaria se puede traducir en el desamparo, la pobreza y discriminación o en la protección, cooperación y sustento de otras vidas (Butler, 2009: 58).

La viabilidad de la vida está determinada e impuesta por diversas normas que nos indican qué vidas deben ser reconocidas, qué vidas pueden habitar su cuerpo y qué muertes pueden ser lloradas (Butler, 2009 b). Para las personas que no responden al criterio heteronormativo, habitar su cuerpo se convierte en algo peligroso; aquellos cuerpos que no corresponden con la norma que se establece en el espacio social y político, corren el riesgo de no tener ninguna significación. 
Reflexionar en torno a la posibilidad de la vida es repensar la normatividad, cuestionarnos por qué ciertas vidas son posibles, son concebibles desde nuestros marcos de entendimiento, y otras no (Butler, 2012: 310). Poder reconocer a las comunidades de personas invisibilizadas por los marcos de inteligibilidad requerirá enfrentarse y trasgredir el marco, proponiendo y actuando de forma distinta a él (Castro, 2014).

Proyectos como el de «Reapropiarnos de nuestras propias historias» pretenden, justamente, confrontar los marcos de entendimiento al visibilizar narrativas de vidas de mujeres lesbianas, bisexuales y pansexuales que están fuera de la normatividad. Mediante la creación de discursos alternativos, tanto textuales como visuales, uno de los objetivos es habitar el espacio público para que los cuerpos invisibilizados por la heteronormatividad puedan ser reconocidos.

\section{Las historias de vida como herramienta de reapropiación del espacio público}

El proyecto de Reapropiándonos de nuestras vidas pretende dar voz a aquellas personas que la investigación más tradicional en ciencias sociales ha silenciado a lo largo del tiempo. Como en otros proyectos donde nos hemos involucrado (Aguirre et al., 2012), no pretendemos convertirnos en portavoces de las protagonistas de los relatos, ya que no lo necesitan, pues ellas mismas tienen la capacidad de expresar y decir aquello que quieren compartir y cómo lo quieren hacer.

Acogemos con fuerza nuestro proyecto como un proceso de empoderamiento, una posibilidad de cambio y visibilización. Y que de acuerdo con Rappaport (1984), marcamos nuestra idea de empoderamiento como un proceso de transformación personal, pero también colectivo, mediante el cual las personas fortalecen sus capacidades, la confianza, la visión y el protagonismo para impulsar cambios positivos en las situaciones que viven. Ellas son las verdaderas protagonistas de sus vidas, las generadoras de cambios y las que posibilitarán la esperada transformación. Porque empoderar implica un cambio de actitud: una nueva predisposición a la acción (Traver, 2009). 
Por otro lado, no podemos abandonar la idea de que el empoderamiento, tal y como lo concebimos, está directamente relacionado con el aprendizaje a lo largo de la vida. Ya que éste «incluye todas aquellas acciones educativas que favorecen y potencian el desarrollo personal, social y profesional de las personas» (Domènech, 2010). Creemos en el aprendizaje a lo largo de la vida como un proceso que se da en el día a día, sin tener que acotarse a la educación reglada y, por ello, centramos nuestro trabajo en la educación informal. Ésta se considera como la modalidad de educación omnipresente y, podría ser, a la larga, la más importante de todo el panorama educativo (Coombs, 1985). Envolvemos dentro de informal toda aquella educación más cercana a la vida, cercana a esa vida que te permite empoderarte con cada experiencia y con ello, transformarte y actuar para promover el cambio.

Desde esta visión, las historias de vida se convierten en una herramienta fundamental de empoderamiento personal y de transformación colectiva. Las personas dan forma a sus vidas cotidianas por medio de los relatos sobre quiénes son ellos y los otros conforme interpretan su pasado en función de su historia, esta reapropación de su vida es, por sí misma, un elemento facilitador del cambio.

Por otra parte, tal y como dice Blanco (2011), es posible leer una sociedad a través de una biografía, los relatos son artefactos sociales que nos hablan tanto de una sociedad y una cultura como lo hacen de una persona o un grupo. La idoneidad de utilizar esta metodología reside en lo que Booth (1998) denomina «la tesis de la voz excluida». Según este autor, los métodos narrativos facilitan el acceso a los puntos de vista y experiencias de los grupos oprimidos que carecen del poder de hacer oír sus voces a través de los sistemas tradicionales del discurso académico. Una segunda razón para ello es la crítica a la erudición tradicional por haber subordinado la realidad de la vida de las personas a la búsqueda de la generalización, mediante la cual se pierde precisamente el detalle que distingue las experiencias personales.

\section{El taller: Reapropiándonos de nuestras vidas}

Como hemos explicado, este proyecto tiene como eje empoderar y visibilizar a las mujeres lesbianas, bisexuales y pansexuales a partir de la metodo- 
logía de historias de vida. Para ello, se planificaron y realizaron en la ciudad de Barcelona dos talleres, de cinco sesiones cada uno, con grupos de mujeres.

La primera sesión estuvo centrada en el río de la vida. La actividad pretendía hacer una primera foto del recorrido personal a través de la metáfora del río que nace y fluye con más o menos caudal, por lugares anchos o estrechos, por paisajes frondosos o desérticos, subiendo montañas o bajando en cascada. A lo largo de la sesión, las participantes dibujamos nuestro río particular con el objetivo de comenzar a hacer visibles su historia, los momentos que más relevancia han tenido para nosotras y los ciclos vitales que hemos atravesado. Comenzábamos la aventura de mirar nuestro recorrido y la interpretación que actualmente hacemos del pasado y del presente.

Siguiendo con ese trabajo, en la segunda sesión reflexionamos sobre tres etapas fundamentales en la configuración de nuestra forma de relacionarnos afectiva y sexualmente con los demás: la infancia, la adolescencia y la actualidad. Para que pudiéramos trabajar sobre aspectos concretos de cada uno de estos momentos vitales, las participantes trajimos tres fotos significativas, una de cada etapa. A partir de esas fotografías, cada una se centraba en momentos concretos que marcaron su vida y su forma de ver y relacionarse con el mundo.

En la tercera sesión nos encontrábamos en el ecuador del taller. Ahora se trataba de escoger la historia concreta que cada una quería contar para comenzar a elaborar su relato. Con la idea de inspirar y aterrizar los temas del relato, elaboramos unas cartulinas con palabras clave como afectividad, deseo, intimidad, expresión de género patriarcado, activismo, capitalismo, amistad, familia, roles de género o amor romántico. La idea era que pudiéramos contar un momento significativo de nuestra vida relacionado con la experiencia como mujer lesbiana, bisexual o pansexual y que en el relato se pudieran hacer visibles tanto la violencia cultural y exclusora del sistema heteronormativo como las estrategias y capacidades que nos han permitido transformar esas opresiones. Una vez las participantes tuvimos claro nuestra historia, nos dividimos en grupos de tres para contarnos las unas a las otras nuestro relato. Mientras una realizaba una narración oral de su historia, otra escuchaba activamente y la tercera tomaba notas para poder escribir la historia que le estaban contando. La idea era que pudiéramos comenzar a estruc- 
turar su narración y pudiéramos observar qué información faltaba o sobraba, qué partes no se entendían, qué partes emocionaban y qué interpretación de esa historia nos devolvían nuestras compañeras. Al final del taller, cada una se llevó las anotaciones de la historia de otra para escribirla durante la semana y devolvérsela a la protagonista. Este primer escrito serviría de base para el relato autobiográfico.

En la cuarta sesión, las participantes devolvimos en forma de redacción la historia a sus protagonistas. Reflexionamos sobre qué y cómo transmitimos lo que queremos y sobre los elementos que queríamos visibilizar o aclarar de nuestros relatos. Volvimos a debatir sobre la relevancia de contextualizar los textos para hacerlos más comprensibles, para poder identificar qué elementos están relacionados con lo social, lo cultural o lo estructural, y de qué forma ha afectado a nuestras vivencias haber crecido en una sociedad capitalista, patriarcal y heteronormativa. También apuntamos lo necesario que es destacar las estrategias, alianzas y capacidades que cada una ha puesto en juego para poder transformar los límites con los que se ha encontrado.

En la quinta sesión, las participantes teníamos nuestro relato elaborado y aprovechamos para hacer una lectura colectiva de los mismos. Esta última sesión estuvo dirigida especialmente a revisar el estilo y la forma en que las participantes queríamos presentar nuestros relatos: texto, audiovisual, fotografía... y, así, comenzar a preparar la segunda fase del proyecto, centrada en la elaboración, a partir de los relatos de vida, de un material didáctico sobre la diversidad sexual dirigido al estudiantado de educación secundaria.

Esta sesión también sirvió para realizar una evaluación colectiva del taller. Entre las cuestiones que se valoraron más positivamente fue el clima de respeto e intimidad que se generó en el espacio del taller. Desde el primer momento pactamos unos principios básicos de funcionamiento del grupo: confidencialidad, horizontalidad, respeto mutuo y comunicación sincera y, como grupo, conseguimos que estos principios se mantuviesen y pudiera crearse un espacio de cuidado, confianza, seguridad e intimidad fundamental para poder compartir nuestra vida.

Por todo lo expuesto, podemos apuntar que las cinco sesiones del taller de Reapropiándonos consiguieron los dos objetivos clave marcados al inicio del proyecto. Por una parte, visibilizamos nuestras historias como mujeres les- 
bianas, bisexuales y pansexuales a partir de la elaboración de nuestros relatos de vida, algo que también nos permitió reapropiarnos de nuestra vida y conectarnos con nuestra capacidad de agencia. Por otra parte, el propio espacio del taller se convirtió en un lugar de reconocimiento, cuidado, seguridad y afecto que, en sí mismo, acabó siendo un lugar de empoderamiento personal y colectivo gracias a la fuerza del propio grupo.

\section{Siguiente paso... la prevención del bullying LGTBIQ+ fóbico}

Como hemos apuntado, el proyecto no termina aquí. Las participantes del taller ahora nos embarcamos en otra aventura: llevar nuestras historias a las aulas de secundaria para introducir la diversidad sexual en la educación formal.

Los estudios y estadísticas recientes muestra que, a día de hoy, uno de los motivos que más pie da a ser víctima de acoso escolar o bullying es la homosexualidad. A los hombres y a las mujeres se le atribuyen unas características que configuran, a su vez, roles sociales inflexibles, de manera que la sociedad puede llegar a condenar, bajo la forma de insultos, agresiones físicas o sexuales, o aislamiento, a aquellas personas cuyos comportamientos o actitudes excedan lo que se ha esperado históricamente de un hombre o de una mujer (Platero y Gómez, 2007).

Es por esto que esta nueva etapa nos llena de emoción. No sólo porque cuando éramos adolescentes nos hubiese encantado que la visibilización de opciones fuera de la heteronormatividad estuviera presente, sino porque creemos fundamental seguir trabajando para que todas las personas puedan gozar de espacio, de reconocimiento y de una vida vivible.

\section{Referencias}

Aguirre, A. ex A $\boldsymbol{A}$. (2012): «Relatos de Vida. Una forma de aprendizaje y empoderamiento personal», II Congreso Internacional de Aprendizaje a lo largo de la vida, Universitat Jaume I, Castelló.

Blanco, M. (2011): «Investigación Narrativa: Una forma de generación de conocimiento», Argumentos, 24(67): 135-156.

Воотн, T. (1998): «El sonido de las voces acalladas: cuestiones acerca del uso 
de los métodos narrativos con personas con dificultades de aprendizaje». En Barton, L. (Comp.): Discapacidad y sociedad, Morata, Madrid.

Butler, J. (2009): Vida precaria. El poder del duelo y la violencia, Paidós, Buenos Aires.

ButLer, J. (2009 b): Dar cuenta de uno mismo. Violencia ética y responsabilidad, Amorrortu Editores, Buenos Aires.

Butler, J. (2012): Deshacer el género, Paidós, Barcelona.

Castro, X. (2014): La propuesta de responsabilidad moral de Judith Butler desde la Filosofía para hacer las Paces. Tesina de Máster Universitario en Estudios Internacionales de Paz, Conflicto y Desarrollo, Universitat Jaume I, Castellón (inédito).

Coombs, P. (1985): La crisis mundial de la educación, Santillana, Madrid.

DoMÈNECH, V. (2010): «Competencias para toda la vida y compromiso docente». En Martín, M. y M. L. SAnchiz (eds.): El aprendizaje a lo largo de la vida: una propuesta de futuro, Ediciones del CReC, Xàtiva, pp. 51-91.

Platero, R. y E. Gómez (2007): Herramientas para combatir el bullying homofóbico, Talasa Ediciones, Madrid.

RAPPAPORT, J. (1984): «Studies in Empowerment: Introduction to the Issue». En Rappaport, J.; C. Swift y R. Hess (eds.): Studies in Empowerment: Steps Toward Understanding and Action, The Haworth Press, New York, pp. 1-7.

Traver, J. A. (2009): Una estrella dansaire. Projecte Docent. Departament d'Educació en l'àrea de Teoria i Història de l'Educació. Universitat Jaume I, Castelló (inédito). 